\title{
REMARQUES SUR LA CULTURE DES PARASITES
}

\section{DU PALUDISME}

\author{
Par A. HOROVITZ et J. SAUTET
}

\section{I. - Historique}

Laveran fut le premier à obtenir la conservation des Plasmodium pendant plusieurs jours. On lit en effet, dans son Traité du paludisme, qu'il a conservé en goutte pendante, pendant 10 jours, du sang de paludéen et qu'il a remarqué pendant ce temps des élèments parasitaires au milieu d'hématies à peine altérées. Mais l'auteur estime ne pas avoir eu de culture, car il lui a été impossible de constater clairement, soit une reproduction, soit même une évolution.

Coronado et Caspar Miller, en 1891 et 1894, disent avoir réussi la culture des Plasmodium, mais cette affirmation n'est pas fondée. En 1897, H. Vincent conserve entre lame et lamelle du sang de paludéen, à la température du laboratoire ou à $32^{\circ}$. Au bout de 8 et 22 heures, il constate la présence de parasites dans les leucocytes.

La question en était là quand Bass, puis Bass et Johns en 1912 déclarèrent avoir cultivé les trois espèces de Plasmodium du paludisme. Après un certain nombre d'essais ils 's'arrêtèrent à une technique assez simple, qui variait suivant que l'on voulait obtenir une seule ou plusieurs générations de parasites. La culture était faite avec du sang défibriné ou citraté porté en anaérobiose à $40^{\circ}$; la décomplémentation du sérum leur paraissait nécessaire, ainsi que l'adjonction de dextrose. Pour cultiver plus d'une génération, il fallait centrifuger le sang afin d'enlever les leucocytes. Les auteurs indiquent dans leurs publications une foule de détails sur la technique et depuis leur travail, rien d'important n'a été ajouté. C'est une méthode assez délicate et donnant des résultats médiocres comme toutes les autres.

Aussitôt ce travail connu, de nombreux auteurs essayèrent de le vérifier et reproduisirent ces « cultures » avec un succès plus ou moins partiel. Citons les travaux de Sinton en 1912, de J.-G. Thomson et Mac Lellan, de J.-G. Thomson et D. Thomson, de

Annales de Parastrologie, T. VII, $\mathrm{N}^{0} 2 .-1^{\text {er }}$ mars 1929 , p. 151-160. 
Ziemann, de Rocha-Lima et Werner, d'Ed. et Et. Sergent, Béguet et Plantier, de Martini, de Lavinder et d'autres en 1913 et 1914. Toutes ces publications apportent (si l'on fait exception pour celle de Sinton) une confirmation des résultats obtenus par Bass et Johns, tout au moins en ce qui concerne la culture d'une seule génération de parasites. Quant aux repiquages, ceux qui ont pu les reproduire, sont rares et les ont obtenus d'une facon exceptionnelle, qu'il importerait de discuter. De toutes ces expériences, il résulte que les points de détail donnés par les premiers auteurs ne sont pas tous absolument nécessaires. C'est ainsi que la température de culture peut être très variable, allant de $25^{\circ}$ à $41^{\circ}$. Par contre, tout le monde est unanime à reconnaître qu'il est nécessaire d'ajouter quelques gouttes d'une solution de dextrose au sérum de culture et que le contact de l'air avec le sang parasité est défavorable au développement des hématozoaires. De plus, l'évolution des parasites est toujours intraglobulaire et la culture du Plasmodium falciparum est la moins difficile à obtenir. Tous ces auteurs apportent donc bien peu de perfectionnements à la méthode, et leurs travaux viennent confirmer simplement ceux de Bass et Johns.

Par contre, Joukoff en 1913, et Perekropoff en 1914, empleient des méthodes simplifiées et annoncent des résultats étonnants. Alors que tous les autres chercheurs n'obtiennent qu'une évolution asexuée, ils constatent facilement in vitro une reproduction sexuée allant jusqu'au stade sporozoïte ! Malheureusement, les figures données semblent bien être des erreurs d'interprétation et leurs expériences ne purent jamais être reproduites avec succès.

En 1917, Row propose une modification de la méthode de Bass permettant l'emploi d'une moins grande quantité de sang, mais la façon d'obtenir l'anaérobiose, dans un tube à étranglement servant à la culture sur pomme de terre, nous semble une difficulté de technique, plutôt qu'une simplification. En 1922, Sinton propose à son tour une modification de la méthode de Row, permettant d'opérer sur une quantité de sang encore plus faible. Cette méthode, plus compliquée que toutes les autres, ne donne pas de meilleurs résultats. Donc, après la découverte de la méthode de Bass, on la modifie, puis comme cela arrive presque toujours, sous prétexte de la simplifier on la complique, aussi, en 1922, a-t-on atteint le maximum de difficultés. C'est seulement álors que l'on s'aperçoit que pour avoir d'aussi médiocres résultats, il n'est nullement besoin de recourir à de telles complications, et que la plupart des précautions, si minutieusement observées jusque-là, ne sont pas nécessaires, en particulier l'anaérobiose. Aussi Row, revenant presque entièrement à la méthode de Bass, publie en 1928 une nouvelle 
technique, qui est simple et donne d'aussi bons résultats que les autres.

\section{II. - Technique}

Puisqu'avec toutes les méthodes publiées, les résultats sont à peu près identiques, la méthode la plus simple est donc la meilleure. Nous nous sommes arrêtés à une technique qui est celle de Bass, modifiée par Row en 1928. Nous n'y avons apporté que des changements de détail.

\section{A. Matériel :}

Il est fort simple et comporte un certain nombre de tubes à essai d'un centimètre de diamètre (dont pour quelques-uns, on aplatit le fond par chauffage); une solution de dextrose à 50 p. 100 dans de l'eau distillée; une seringue avec aiguille pour ponction veineuse et une pipette. Le tout doit être rigoureusement stérilisé.

\section{B. Manipulation :}

Elle comprend les temps suivants :

1. - Ponction veineuse d'un paludéen en accès : retirer $10 \mathrm{~cm}^{3}$ de sang.

2. - Répartition de quelques gouttes de sang dans des tubes à essai stériles. On défibrine par agitation rapide de ces tubes.

3. - Le reste du sang est réparti dans d'autres tubes en quantité suffisante pour permettre de recueillir le sérum après coagulation (1).

4. - On laisse le tout à la température du laboratoire pendant quelques heures sans inconvénient. Il importe toutefois que cette température ne soit guère inférieure à $18^{\circ}$ ou $20^{\circ} \mathrm{C}$.

5. - On répartit le sérum du malade à raison d'environ un centimètre cube dans chaque tube à fond plat. On ajoute 1 à 2 gouttes de la solution de dextrose. On ensemence enfin 2 ou 3 gouttes du sang défibriné contenant les hématozoaires. On met alors à l'étuve à 35 ou $38^{\circ}$. On peut même laisser à la température du laboratoire, si elle

(1) On peut se servir aussi, pour la culture, de sérum préalablement recueilli chez un autre homme et ainsi préparer des tubes d'avance avec du dextrose. On ensemence dans ce cas immédiatement après la défibrination du sang du malade et l'on porte à $35^{\circ}$ ou $37^{\circ}$. Mais nous recommandons plutôt l'emploi du sérum du malade lui-même. 
n'est pas inférieure à $25^{\circ}$; l'évolution sera seulement moins rapide.

6. - On examine les cultures après $24,48,72$ heures. Pour cet examen, on se sert d'une pipette effilée, moyennement fine, dont la

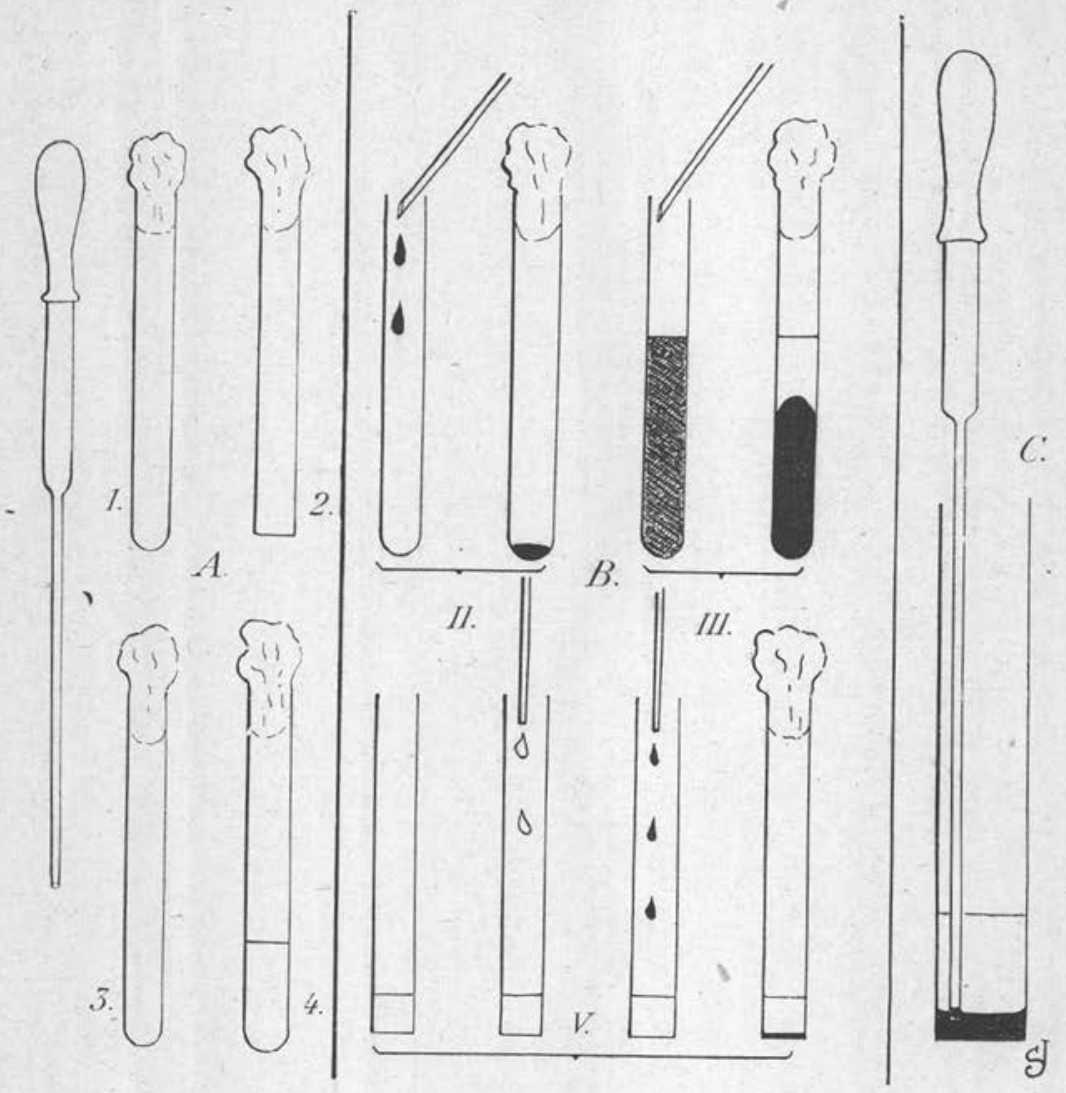

FIG. - A. - Matériel : 1, tube à défibriner le sang; 2, tube de culture ; 3 , tube à coaguler ; 4, solution de dextrose à 50 p. 100 . B. - Manipulation : II, III, V, temps $2,3,5$ du texte. C. - Examen d'un tube de culture : prélèvement à la surface de la couche d'hématies.

section sera bien régulière, de façon à faire un prélèvement très exactement à la surface de la couche de globules rouges. On fait alors des frottis comme pour du sang ordinaire et on les colore par les mêmes méthodes.

On constate que les parasites se trouvent uniquement à la surface de la couche des hématies et qu'il n'y en a jamais dans le sérum. 
Il nous semble inutile de décomplémenter le sérum que l'on emploie. Pour que les cultures soient riches, il est nécessaire que le malade ait eu au moins 4 ou 5 accès et que le sang soit prélevé au moment où le malade est en plein accès.

Le sérum laqué est à rejeter, il ne donne que des échecs.

\section{III. - Discussion}

Une question se pose. A-t-on une reproduction certaine des parasites avec envahissement de nouvelles hématies ?

Bass et Johns affirment qu'ils ont eu des cultures véritables, tout en reconnaissant les imperfections de leur méthode. Pour eux, les parasites asexués augmentent de volume, se segmentent et donnent naissance à des mérozoites, dont beaucoup pénètrent dans de nouvelles hématies. La plupart de leurs expériences ont donné lieu à une ou deux générations. Dans un cas ils ont même pu avoir 4 générations. Pour Ziemann, les conclusions sont à peu près identiques, il fait remarquer que le cycle sexué n'existe pas in vitro, que les jeunes mérozoites ne sont que peu de temps libres et que les parasites d'un globule rouge ne le quittent jamais pour aller en parasiter un autre. Il constate aussi le développement complet des parasites dans les hématies, avec apparition de granulations de Schüffner ou de Maurer, comme dans le sang circulant. Mais il fait remarquer aussi qu'il n'est pas possible de cultiver indéfiniment ces parasites sans revenir au cycle sexué.

Connal et Coghill obtiennent dans un cas le développement du Plasmodium falciparum jusqu'au début de la troisième génération. Par contre, d'autres auteurs, comme Sinton en 1912, ne constatent aucune augmentation en nombre des parasites. Lavinder en 1913 observe, dans le cas du Plasmodium falciparum, l'évolution des schizontes jusqu'aux mérozoites, mais il n'a jamais vu de parasites infecter de nouveaux globules rouges. De même Martini en 1914 n'observe qu'une génération. Plus tard Row, après une étude approfondie de la question, conclut à l'impossibilité du repiquage par sa méthode.

Ed. et Et. Sergent, Béguet et Plantier constatent que par la méthode de Bass, on peut avoir l'évolution complète d'une génération de Plasmodium falciparum, depuis la forme annulaire jusqu'au stade mérozoite. Si le prélèvement est fait au stade de jeunes schizontes, on peut avoir in vitro l'infection de nouveaux globules rouges par de très jeunes parasites : c'est ce qu'ils observèrent dans 
un cas : "Ceux-ci semblent bien être les représentants d'une nouvelle génération. " A part cette unique observation, ils n'ont jamais vu d'évolution portant sur plus d'une génération. Après da RochaLima et Werner, ils signalent un fait de la plus haute importance pour l'interprétation des «cultures »: «A côté de nombreuses formes évoluant nettement, un certain nombre restaient stationnaires plusieurs jours, sans apparence de développement, ni de dégénérescence... La survivance de petites formes pourrait en imposer pour l'apparition de jeunes formes de nouvelles générations, cause d'erreur dont il faut se méfier. \$

De toutes ces observations, que devons-nous conclure ?

1. - La plupart des auteurs ne donnent pas le procédé qui leur a permis de mesurer l'accroissement du nombre des parasites; pourtant cela est important à préciser.

2. - Quand même ils auraient comblé cette lacune, il faut faire remarquer que la technique d'une numération sera toujours très délicate, car la « culture 》n'est jamais homogène, les parasites se trouvent rassemblés seulement à la surface de la couche d'hématies et probablement d'une façon irrégulière ; c'est là encore une des causes d'erreur les plus importantes.

3. - A propos de la persistance de formes jeunes sans évolution, leur existence est assez difficile à démontrer d'une façon absolue. En tous cas, ce facteur rend délicate l'interprétation de formes jeunes dans les hématies : est-ce une génération nouvelle ou une partie non évoluée de l'ancienne génération ?

4. - Dans le sang d'un paludéen, il y a toujours une forme prédominant à un stade déterminé, mais il est vrai aussi qu'à côté, avec un peu de patience, on trouve tous les autres stades, bien qu'en moins grand nombre. Ce facteur gêne considérablement l'interprétation des « cultures » dans le cas de plusieurs générations observées.

5. - Presque tous les auteurs sont d'accord pour constater l'évolution complète in vitro d'une-génération de Plasmodium et souvent la formation de gamètes.

6. - Personne n'a observé de reproduction sexuée dans les « cultures s.

7. - Tous ont observé la phagocytose des parasites in vitro.

8. - Les cas où plusieurs générations ont été cultivées sont toujours isolés. Par exemple, Bass et Johns citent un cas où ils obtinrent 4 générations, mais ils ne donnent pas de détails suffisants sur les raisons qui leur ont permis d'apprécier cette évolution. Depuis 1911, personne n'a obtenu de meilleurs résultats de repiquage que ceux de Bass et Johns, au contraire. 
9. - La culture du Plasmodium falciparum est la plus facile à obtenir, celle du Plasmodium malariæ et celle du Plasmodium vivax sont par contre plus délicates.

10. - La plupart des auteurs n'insistent pas assez, à notre avis, sur les difficultés qu'ils ont pu rencontrer et les échecs qu'ils ont pu enregistrer. Trop souvent, comme toujours, on ne rapporte avec détails que les cas heureux.

Telles sont les conclusions que l'on peut tirer des différentes publications que nous venons d'analyser. Pour nous, nous avons essayé, en reproduisant ces expériences, de nous faire une opinion et de constater si les « cultures » étaient susceptibles d'avoir une importance en pratique médicale pour la conservation du virus paludéen. L'interprétation des résultats se heurte aux difficultés que nous avons signalées.

Nous avons tenté nos « cultures » en partant du sang de 5 paralytiques généraux, inoculés avec du Plasmodium vivax. Nous avons opéré suivant les diverses techniques. Les résultats furent toujours médiocres. Aussi nous nous demandons si nous avons eu des « cultures » vraies avec une reproduction certaine des parasites.

L'examen simple ne nous montre pas une pullulation assez censiaérable des parasites pour imposer d'emblée l'idée d'une culture, comme dans le cas des cultures d'amibes dysentériques. Aussi avons-nous essayé de faire des numérations. C'est certes un procédé assez mauvais, dont nous avons déjà fait la critique. Les chiffres que nous donnons servent seulement à concrétiser un peu une idée et nous apportons toutes les restrictions voulues sur la valeur qu'on doit leur attribuer.

Nous prendrons comme exemple les chiffres obtenus dans une de nos cultures les plus favorables.

\section{Observation :}

X., paralytique général. Sang prélevé par voie intra-veineuse au $5^{\circ}$ accès, au début de cet accès. Défibriné dans de petits tubes à essai, ce sang est réparti en deux portions : l'une, conservée à l'hôpital, est mise immédiatement à $38^{\circ}$ dans des tubes de culture, l'autre est emportée à la Faculté de Médecine (1/2 heure de transport) et est laissée à la température du laboratoire pendant 6 heures (octobre 1928) avant d'ensemencer. Toutes ces cultures ont été faites avec le sérum du malade, donc dans les conditions les plus favorables.

Les cultures faites à l'Hôpital, aussitôt après la prise de sang, se sont développées plus vite que celles faites à la Faculté, mais n’ont pas donné de meilleurs résultats. 
Voici les résultats obtenus à la Faculté :

\begin{tabular}{|c|c|c|c|c|c|c|}
\hline & \multirow[b]{2}{*}{ Cultune } & \multicolumn{5}{|c|}{ PARASITES POUR 100 LEUCOCYTES } \\
\hline & & 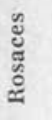 & 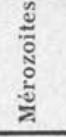 & \multicolumn{2}{|c|}{ Schizontes } & हूँ \\
\hline $\begin{array}{c}\text { Avant } \\
\text { la culture }\end{array}$ & Sang défibriné ......... & 2 & 15 & 21 & & 3 ? \\
\hline \multirow{4}{*}{$\begin{array}{c}\text { Après } \\
24 \text { heures }\end{array}$} & Bass................. & 1 & & 2 & 108 & 4 ? \\
\hline & Row (1917)........... & 0 & 0 & 9 & 116 & $4 ?$ \\
\hline & Row $(1928) \ldots \ldots \ldots \ldots$ & 0 & 0 & 0 & 88 & $6 ?$ ? \\
\hline & Technique décrite p. 153 & 0 & 0 & 1 & 102 & $4 ?$ \\
\hline \multirow{5}{*}{$\begin{array}{c}\text { Après } \\
72 \text { heures }\end{array}$} & Sinton .............. & 0 & 0 & 0 & 76 & $1 ?$ \\
\hline & Bass $\ldots \ldots \ldots \ldots \ldots \ldots$ & 0 & 0 & 0 & 96 & $2 ?$ \\
\hline & Row (1917) ........... & 0 & 0 & 0 & 114 & 4 ? \\
\hline & Row (1928) . . . . . . . . & 0 & 0 & 0 & 121 & $?$ \\
\hline & Technique décrite\{p. 153 & 0 & 0 & 0 & 100 & $3 ?$ \\
\hline
\end{tabular}

Après 72 heures, on ne trouve plus dans toutes ces cultures le parasite typique et bien colorable ; les granulations de Schüffner persistent, mais l'hématie parasitée est altérée ainsi que le Plasmodium lui-même.

La lecture de ce tableau nous montre qu'il ne semble pas y avoir eu accroissement de nombre des parasites ; ceux qui ont été mis dans les tubes de culture ont évolué, mais il ne semble pas que de nouvelles hématies se soient infectés, en partant d'une nouvelle génération développée in vitro. Nous avon̨s seulement trouvé quelques mérozoïtes en libęrté dans le sang, mais il est impossible de savoir ce qu'ils deviennent. Du reste, tous les éléments ont-ils évolué parallèlement ? Il est tout aussi difficile de le savoir, cependant, nous pensons que non, car, dans certaines circonstances peu favorables, nous avons vu des parasites se conserver sans aucune transformation.

Dans les conditions favorables, les hématozoaires évoluent comme dans le sang périphérique. Le stade gamète est souvent atteint.

Done, dans le cas du Plasmodium vivax, nous voyons qu'il n'y a pas de « culture » véritable, puisqu'il n'y a pas une multiplication certaine. C'est plutòt une conservation des parasites, et une conservation trop fugace pour avoir une application pratique quelconque.

Dans le cas des Plasmodium falciparum et malariæ, il semble bien en être de même. De nombreuses et belles préparations, qui 
nous ont été aimablement communiquées par le Docteur Row, confirment notre opinion.

Il ne s'agit donc pas, à proprement parler, d'une culture, et même, si les très rares et peu nombreux repiquages obtenus par quelques auteurs sont bien réels, cette méthode n'en aboutit pas moins à un véritable échec et la culture des parasites du paludisme est encore à découvrir.

Ces travaux ont pourtant été utiles, car ils ont permis d'étudier in vitro l'évolution sexuée des 3 espèces de Plasmodium et de se rendre compte ainsi d'un certain nombre de facteurs l'influençant.

C'est ainsi que la température de culture semble commander le développement, mais il est juste de dire que les conditions d'observation des auteurs sont souvent trop différentes pour qu'on puisse les comparer.

Les réactions sérologiques ont aussi tenté les chercheurs, mais leurs travaux ne sont pas applicables en pratique médicale et sont discutables. De même, le diagnostic du paludisme au moyen des cultures n'est qu'une utopie.

Quant aux études faites sur l'action de la quinine elles sont intéressantes ; malheureusement, les « cultures » ne sont ni assez nettes, ni assez vivaces pour donner une bien grande valeur à ces expériences. Enfin, la simple conservation du virus, qui est la première des qualités d'une culture, est totalement impossible ; en cela, nous en sommes au même point qu'après les premières expériences de Laveran.

\section{BiBLIOGRAPHIE}

Bass (C.-C.). - Successful cultivation of Malarial Plasmodia. Jonrn. Amer. Med. Ass., LVII, 1911, p. 936.

- Cultivation of Malarial Plasmodia in vitro. Amer. Journ. Trop. Dis., I, 1914, p. 546.

Bass (C.-C.) et JoHss (F.). - The cultivation of Malarial Plasmodia in vitro. Journ. Exp. Med., XVI, 1912, p. 567.

Chambellano. - Culture de l'hématozoaire du paludisme. Presse méd., 1919, p. 783.

Connal (A.) et Coghil. (H.). - Bass culture in malaria. Nigeria Annual report., 1915 Coronado et Miller (C.-O.). - Cronica medico quirurgica de la Habana. I, 1891.

Joukoff (M.). - Gultures de Plasmodium prœcox et malariæ. C. R. Soc. Biol., 1913 , p. 136.

LAvinder (C.-H.). - A note on the cultivation of Malarial Plasmodia after the method of Bass and Johns. Journ. Amer. Med. A:s., LX, 1913, p. 42.

Laveran (A.). - Traité du paludisme, 1893.

MARTiNi (E.). - Ueber die Entwicklung von Malariaparasiten im Basschen Nährboden. Centralbl. f. Bakter. Orig., LXXIII, 1914, p. 250.

Mitcer (C.-O.). - Ueber aseptische Protozoenkulturen. Centralbl. $t$. Bakter., XVI, 1894 , p. 132. 
Nöller (W.). - Die Züchtung der parasitischen Protozoen. Handbuch der pathogenen Protozoen, III, 1928, p. 1815.

Peréropoff. - Ueber Kulturen der Plasmodien des tropischen Fieber (Malaria tropica). Arch. f. Protistenkunde, XXXV, 1914, p. 139.

Pewny (W.). - Ueber Malariakulturen. Wien. Klin. Woch., XL, 1917, p. 1358.

Pitschugin (P.). - Kultivierungsversuche mit Plasmodium vivax nach der Methode von Bass. Centralbl, f. Bakter. Origin., LXXIII, 1914, p. 373.

Da Rocha-ima (H.) et Werner (H.). - Ueber die Züchtung von Malariaparasiten nach der Methode von Bass. Arch. f. Sch. u. Trop. Hyg, XVII, 1913, p. 541.

Row (R.). - On a simplified technique of Bass'smethod of cultivating malarial parasites in vitro. Indian Journ. Med. Res., IV, 1917, p. 388.

- A simplified technique for culturing malarial parasites aerobically. Indian Med. Gaz., LXIII, 1928, p. 1.

Observations sur le paludisme et la formation des gamètes de Plasmodium en culture. Bull. Soc. Path. exot., XXI, 1928, p. 607.

Sergent (Ed.), Sergent (Et.), Béguet (M.) et Plantier (A.). - Sur la culture in vitro du parasite du paludisme d'après la méthode de Bass. C. R. Soc. Biol., LXXV, 1913, p. 29.

Sinton (J.). - Some attempts at the cultivation of the malarial parasite by Bass's method. Ann. Trop. Med. and Paras., VI, 1912, p. 371.

- Simplified method for the cultivation of Plasmodium falciparum in vitro. Indian Journ. Med. Res., X, 1922, p. 203.

THomson (D.). - A research into the production, life and death of crescent in malignant tertian malaria, in treated and untreated cases, by an enumerative method. Ann. Trop, med. and Paras., V, 1911, p. 57.

Thомson (J.-G.). - Experiments on the complement fixalion in malaria with antigens prepared from culture of malarial parasites. Proceedings of the Royal Soc. of Med., 1919, XII, p. 39.

Thomson (J.-G.) et Mac Lellans (S.). - The cultivation of one generation of malarial parasites in vitro by Bass's method. Ann. Trop. Med. and Paras., VI, 1912, p. 449.

Thомson (J.-G.) et Thомson (D ). - The cultivation of one generation of benign tertian malarial parasites in vitro by Bass's method. Ann. Trop. Med. and Paras., VII, 1913, p. 153.

- The growth and sporulation of the benign and malignant tertian malarial parasites in culture tubes and in the human host. Ann. Trop. Med. and Paras., VIl, 1913, p. 509.

Vincent (H.). - Le processus leúcocytaire dans la malaria. Ann. Inst. Pasteur, XI, 1897, p. 891.

Ziemann (H.). - Ueber die Bassche Kultur der Malariaparasiten in vitro. Centralbl. f. Bakter. Orig., LXVII, 1913, p. 482.

- Ueber die künstliche Weiterentwicklung (in vitro) des tertian Malariaparasiten. Deutsche med. Woch., 1913.

- Ueber die Kultur der Malariaparasiten und der Piroplasmen in vitro. Arch. für Sch. u. Trop. Hyg., XVII, 1913, p. 361.

On the culture of malarial parasites and Piroplasma canis. Trans. of Soc. Trop. Med. and Hyg., VI, 1913, p. 220.

Weiteres über die Züchtung des Malariaparasiten und der Piroplasmen in vitro. Arch. f. Sch. u. Trop. Hyg., XVIII, 1914, p. 77.

Service du Dr Crouzon à l'Hôpital de la Salpêtrière et Laboraloire de Parasitologie de la Faculté de Médecine de Paris 